\title{
Three-dimensional object constancy: Coherence of a simulated rotating sphere in noise
}

\author{
J. TIMOTHY PETERSIK \\ Southeast Missouri State University, Cape Girardeau, Missouri 63701
}

\begin{abstract}
The maintenance of constant and coherent percepts of three-dimensional objects, even in the midst of visual noise, is important to our ability to navigate the environment. In the present experiments, observers viewed computer-animated simulations of three-dimensional spheres rotating around the vertical axis in depth. In the first experiment, the addition of noise in the form of randomly moving display elements reduced subjects' judgments of depth and the accuracy of their rotation-direction judgments, although the phenomenal appearance of three-dimensional structure was maintained throughout. In the second experiment, a change from frame to frame in the orientation of the vectors that composed the simulation reduced, but did not destroy, perceived depth and rotation-direction accuracy. The effect of a change in orientation between vectors of successive frames of a simulation depended upon the length of those vectors. It is argued that dynamic perspective- the information provided by movement and perspective together-is a significant factor in the maintenance of threedimensional object constancy.
\end{abstract}

Despite the complex flow of information across the retina that results from object movement and selfmovement in the environment, we rarely experience any difficulty in the maintenance of a coherent and constant percept of an object or in the identification of many of its secondary attributes (e.g., its direction of movement or rotation, its size, its extent in depth). Although the conditions under which three-dimensional object constancy occurs are not yet fully specified (cf. Epstein, 1977), it has been well established that two sources of information, namely motion and perspective, greatly enhance coherent percepts of constancy in depth (Braunstein, 1976; Johansson, 1974, 1977). Together, motion and perspective information constitute what can be called dynamic perspective, that is, the continuous or discrete perspective transformations (cf. Gibson, 1957) of the two-dimensional projection of an object as a function of its movement (e.g., rotation) in the visual field. The present paper examines some of the minimal conditions of dynamic perspective transformations that permit object con-

\footnotetext{
These studies were completed in partial fulfillment of the requirements for the doctoral degree at Miami University, Oxford, Ohio. Both experiments were presented at the 19th annual meeting of the Psychonomic Society in San Antonio, Texas, November 1978. The author gratefully acknowledges the guidance of Allan Pantle in all stages of this project. Marvin Dainoff, John Jahnke, Richard Sherman, and Jim Malott each contributed to the development of these studies. Special acknowledgement is due the observers who maintained an interest in this research and who continued to volunteer their time throughout its duration. Requests for reprints should be addressed to J. Timothy Petersik, Department of Psychology, Southeast Missouri State University, Cape Girardeau, Missouri 63701.
}

stancy, and it is concluded that dynamic perspective information can lead to coherent percepts of objects that are maintained amidst relatively high levels of noise.

Subjects judged the apparent rotation direction and apparent depth of computer-animated simulations of a transparent sphere partially filled with randomly positioned luminous dots or vectors and rotating around the $\mathrm{Y}$-axis in depth. Noise was introduced to the displays by (a) varying the ratio of display elements that moved according to the rules of perspective (signal dots) to those that moved randomly (noise dots), or (b) changing the orientation of signal vectors from frame to frame of a simulation.

\section{Computer Displays}

A modification of Braunstein's (1976) method for simulating the rotation of a sphere in depth was used. Briefly, a three-dimensional space corresponding to the volume of a sphere is established in the memory of a computer, and display elements are randomly positioned at fixed locations within that volume. Next, the two-dimensional projection of that volume is created and stored in computer memory in the form of a subpicture (1). The "sphere" is then symbolically rotated by a specific angle, a new twodimensional projection is determined, and Subpicture 2 is stored. This process continues until the sphere has been rotated through $360^{\circ}$ and a set of corresponding subpictures has been stored. Then the sequence of subpictures is displayed either in a forward order, simulating a clockwise rotation of the sphere, or in a backward order, simulating a counterclockwise rotation. 
All displays were constructed by a PDP-11/10 computer and were presented on-line by the same computer on an oscilloscope coated with a rapiddecay phosphor (P31). During the experiments, the values of the parameters used in the construction and display of the rotation simulation were stored on a magnetic disk. Different sets of these parameters, corresponding to different experimental conditions, were accessed by the computer as it ran the experiment. The most important of these parameters were: (1) Simulated projection distance, or the assumed distance from the center of the rotating sphere to the observer, expressed in terms of the number of sphere radii contained in that distance. Small values produce polar projections and contain much perspective. Large values approach a parallel projection and have little perspective. (2) Number of frames in a $360^{\circ}$ cycle of rotation and, correspondingly, the angle of rotation between successive frames. (3) Number of display elements within a sphere. (4) Frame duration. (5) Interstimulus interval, or interframe interval (ISI).

The positions of the component elements in each simulated sphere were independent of the positions of elements in any other simulation. Random numbers corresponding to experimental conditions were manually entered; otherwise each of the experiments was controlled by the computer.

Since the volume of any sphere was filled randomly with elements, spheres were never of a constant angular size objectively. However, the dynamic perspective transformations of the dots established a kind of "perceptual boundary" or surface of the apparently rotating sphere. In most cases, this subjective boundary corresponded to what is hereafter referred to as "maximum visual angle." Operationally, maximum visual angle is the subtense of any sphere when that sphere is completely filled with elements. In fact, maximum visual angle was determined by creating one frame of a sphere simulation with a very high element density and then measuring its diameter.

Petersik (Note 1) has shown that, when no noise is present in these displays, simulations composed of as few as 10 elements in each of 25 frames per $360^{\circ}$ of rotation yield striking percepts of constant objects rotating in depth. Generally speaking, the more display elements and the more frames, the more convincing is the simulation. Provided that the simulation employs a polar projection, the direction of rotation of the simulated sphere is relatively unambiguous. With no noise present, subjects can report the rotation direction of each simulation with $65 \%-90 \%$ accuracy, depending upon the amount of perspective present and the values of other display parameters. In addition, the subjective impression of most subjects is that the "front" and "rear" surfaces of the rotating sphere can be perceived along with the volume of the sphere.

\section{EXPERIMENT 1}

\section{Method}

Subjects. Eight psychology students volunteered to participate in the experiment. All subjects reportedly had $20 / 20$ visual acuity, either with corrective lenses or without them. Five of the eight subjects had previously served in a similar experiment. However, the shapes of the functions yielded by each of the five experienced subjects were similar in appearance to those yielded by the inexperienced subjects.

Stimuli. Display elements were dots. Each dot subtended 1.5 visual angle and had a luminance of $4.39 \mathrm{~cd} / \mathrm{m}^{2}$. The mean luminance of a simulated sphere was $.03 \mathrm{~cd} / \mathrm{m}^{2}$. The maximum visual angle subtended by the diameter of any sphere was $2.5^{\circ}$.

The values of display parameters held constant were (a) dot numerosity, at 45 dots, (b) frame duration, at $100 \mathrm{msec}$, (c) ISI, at $15 \mathrm{msec}$, and (d) number of complete rotations per trial, at two. The direction of rotation on any trial was randomly determined by the computer, with a .5 probability of clockwise rotation.

Procedure. Each subject served in eight sessions, one practice session and seven experimental sessions, each held on a different day. Displays were viewed binocularly and without a fixation point, except for a 3-sec-long blinking warning light which preceded each trial and which appeared at the location corresponding to the center of the sphere. Each subject sat directly in front of the display oscilloscope at a distance of $200 \mathrm{~cm}$. The oscilloscope was approximately at eye level. Each display was viewed in a nearly dark room. Slight ambient illumination was produced by a night-light which was used to illuminate the computer Teletype between trials. Each subject was dark-adapted prior to an experimental session.

Before any subject served in an experimental session, he served in a 50-min-long practice session. There were three purposes for the practice session: (1) to acquaint the subject with the displays and experimental procedure; (2) to provide the subject with feedback regarding the correctness of his initial rotation-direction judgments and thereby reduce any potential for response bias in later sessions; and (3) to present the subject with a range of exemplary stimuli that served as anchors for the subject's depth ratings.

During a single experimental session, a subject viewed one block of 48 different displays (trials) resulting from the factorial combination of two frame numerosities (10 or 50 frames per $360^{\circ}$ rotation), four projection distances $(1.2,5.0,15.0$, or 99999.0 sphere radii), and six percentages (numbers) of signal dots-9\% (4 dots), $20 \%$ ( 9 dots), $40 \%$ (18 dots), 60\% (27 dots), 80\% (36 dots), and $91 \%$ (41 dots). The order of presentation was random. After each trial, the subject reported (1) the perceived direction of rotation of the sphere, and (2) the perceived "depth" of the sphere, a concept that included its "volume"' and "goodness," on a scale from 0 (no depth) to 6 (much depth). Fractional values were allowed, although they were used infrequently. No feedback was provided after a trial. Approximately $20 \mathrm{sec}$ elapsed between trials.

\section{Results and Discussion}

Direction of rotation judgments. Figure 1 shows, for each of the three polar projections used in the experiment, the percentage of correct rotation-direction judgments, collapsed across the number-of-frames factor, as a function of the percentage of signal dots. The solid horizontal line that crosses the ordinate at the $50.3 \%$ value is the mean percentage of correct rotation judgments obtained in the parallel-projection conditions, i.e., the 99999.0 sphere-radii projections. The data summarized by the horizontal line replicate past findings (reviewed in Braunstein, 1976), which have shown that observers cannot accurately discriminate rotation direction in parallel-projection dis- 


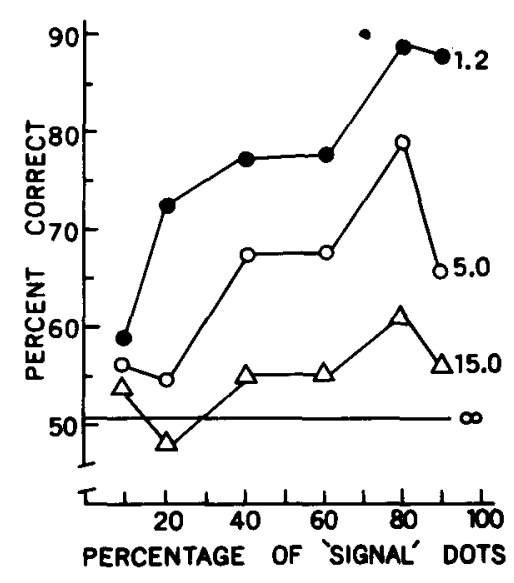

Figure 1. Percentage of correct rotation-direction judgments, collapsed across number of frames per trial, as a function of the percentage of dots that were transformed according to the rules of perspective (signal dots). Each curve depicts the effect for a different projection distance (in sphere radii). The solid horizontal line through $50.3 \%$ is the mean percentage of correct judgments for all paraltel projections.

plays. Such findings are to be expected, since parallel projections do not contain information about rotation direction (cf. Braunstein, 1976). In such displays, although the subject perceives a rotating sphere, the direction of rotation is ambiguous and perceptually switches from time to time. Statistical analysis confirms the impression gained from Figure 1 that projection distance had a significant effect on the percentage of correct rotation-direction judgments $[F(3,21)=31.70, p<.001, S E=2.56]$ in a repeated measures analysis of variance. That is, the curve for the 1.2 sphere-radii polar-projection conditions lies above the curve for 5.0 sphere-radii polar-projection conditions, etc.

In addition, there was a significant main effect of percentage of signal dots $[F(5,35)=5.60, p<.001$, $\mathrm{SE}=2.56]$. In general, for each projection distance, as the percentage of dots transformed according to perspective rules increased, the percentage of correct rotation-direction judgments also increased. However, there was also a significant interaction of projection distance and percentage of signal dots $[F(3,21)$ $=4.98, \mathrm{p}<.01, \mathrm{SE}=3.16$ ). The form of the interaction can be seen in Figure 1, where the slope of the curve relating percentage of correct rotation-direction judgments to percentage of signal dots is greatest for the 1.2 sphere-radii projections $(m=.32$, leastsquares fit), slightly shallower for the 5.0 sphereradii projections $(m=.21)$, and greatly reduced for the 15.0 sphere-radii projections $(\mathrm{m}=.09)$.

There was no effect of number of frames on the percentage of correct rotation-direction judgments $[F(1,7)=2.03, p>.05]$. Thus, subjects were just as accurate in the determination of rotation direction when a cycle of rotation was depicted by only 10 frames as when it was depicted by 50 frames. This result confirms an earlier finding of Petersik (Note 1). When successive "glimpses" of a rotating object are separated by as much as $36^{\circ}$ of rotation, subjects can determine rotation direction as accurately as when the successive glimpses are separated by only $7.2^{\circ}$ of rotation. This feat suggests a remarkable sensitivity of the visual system to three-dimensional dynamic perspective transformations.

Depth judgments. Figure 2 shows, for each of the two frame numerosities used in the present experiment, the mean depth rating produced in response to the rotating spheres as a function of the percentage of signal dots within the displays. As is apparent in the figure, depth ratings increased with successively greater increases in the percentage of signal dots as well as with an increase in the number of frames. In a repeated-measures analysis of variance, frame numerosity had a significant main effect on mean depth judgments $[F(1,7)=36.44, p<.001$, SE $=$ $.11]$, as did percentage of signal dots $[F(5,35)=$ $39.10, \mathrm{p}<.001, \mathrm{SE}=.12$ ]. It could be argued that the increase in rated depth with increases in the percentage of signal dots is due to the greater probability with larger percentages of signal dots that more dots in the periphery of the sphere will be transformed according to the rules of perspective. This argument can probably be rejected for two reasons. First, depth ratings were not based upon the size of the rotating sphere, but rather upon the sensation of volume elicited by the sphere as well as upon the sphere's "goodness," a factor that included the detectability of the front and rear surfaces of the sphere. Second, the percentage of signal dots was also shown to affect the percentage of correct rotation-direction

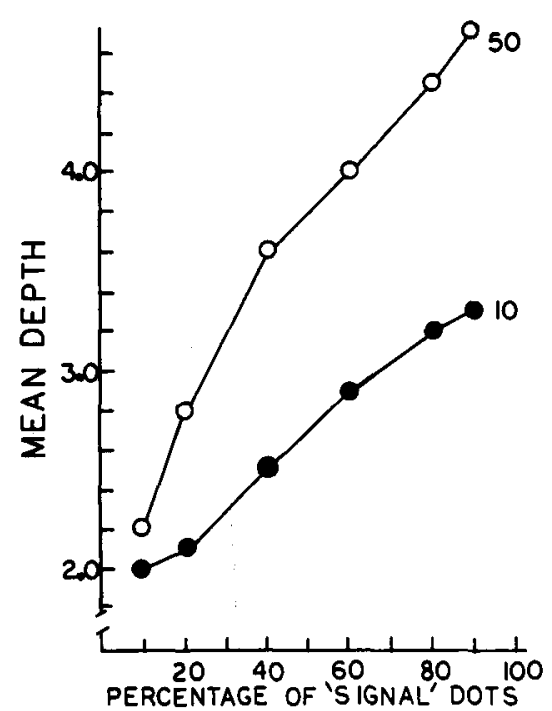

Figure 2. Mean depth ratings, collapsed over projection distance, as a function of the percentage of signal dots. Each curve depicts the effect for a different frame numerosity. 
judgments; the perception of rotation direction is presumably not affected by the location within the sphere of the perspective-transformed dots as long as they are uniformly distributed. Therefore, it is likely that the effects described here are mediated by the greater sensitivity of the observer to the perspective transformations per se with increases in the percentage of signal dots.

There was also a significant interaction of frame numerosity and percentage of signal dots $[F(5,35)=$ $9.90, \mathrm{p}<.001, \mathrm{SE}=.08]$. As shown in Figure 2, the effect of percentage of signal dots was greater for the 50 -frame displays than for the 10 -frame displays; i.e., the slope of the 50-frame function is steeper $(\mathrm{m}=.03$, least-squares fit) than the slope of the 10-frame function $(m=.02)$. Although there were significant effects of frame numerosity and percentage of signal dots, none of the displays viewed in the present experiment failed to elicit some sensation of depth. That is, the average depth ratings assigned to these displays were above 2.0 . This fact does not reflect a bias on the parts of observers against using the lowest depth ratings, since Petersik (Note 1) has shown that some rotation simulations of this type do appear flat and subsequently elicit appropriate depth ratings. The finding that even displays with only $9 \%$ signal dots and consisting of only 10 frames produce some sensation of depth reinforces the conclusion that the visual system is especially sensitive to dynamic perspective transformations.

\section{EXPERIMENT 2}

Another method of introducing noise in the present displays is to vary over time the similarity of the elements that compose the simulation of the rotating sphere. Thus, in the present experiment, display elements were vectors rather than dots. Vectors oriented vertically appeared in the first, third, fifth, and each successive odd-numbered frame of a display sequence. Vectors in the even-numbered frames were shifted from vertical by a specific angle between $0^{\circ}$ and $65^{\circ}$. Any given vector was shifted either clockwise or counterclockwise from vertical, and the direction of the orientation change was randomly determined for each vector in each even-numbered frame. For any simulation, each vector in the evennumbered frames was shifted by the same angle as every other vector. The orientation change was relative to the center of the vector's position in threedimensional space.

Using stereoscopic, rather than movement, displays, Frisby and Julesz (1975) have recently conducted an analogous type of experiment. One member of a stereographic pair had line segments that were oriented horizontally. The orientation of the line segments in the second member of the pair varied from trial to trial. In addition, line length varied across trials. Within each member of a stereographic pair, there was a central square area composed of 16 lines that were positioned to produce a crossed disparity of $4^{\prime}$. The authors found that the perceived depth of the disparate central square decreased with increases in the vertical distance between the projection of the endpoint of a line segment on one retina and the projection of the endpoint of its corresponding line segment on the other retina-a joint function of line length and the difference between the orientations of the two lines. Frisby and Julesz termed the vertical distance between the endpoints of corresponding line segments vertical disparity.

In movement displays of the kind described here, depth percepts are not established by spatial disparities between inputs to the two eyes. Rather, the sensation of depth seems to be produced by a comparison of the positions of the corresponding elements of the display over time. Nonetheless, we might expect that there would be some similarity in the response of the movement-detecting system to the response of the stereoscopic system when analogous types of stimuli are presented. Therefore, vector length and vector orientation between frames were both varied in the present experiment in an attempt to create stimuli that were temporally analogous to the stereograms used by Frisby and Julesz. If the process that organizes the sphere simulation into a coherent whole behaves like the stereoscopic process that segregates correlated but disparate areas in random-line stereograms, orientation difference and vector length should have significant effects on the ability of a subject to perceive the direction of rotation of a sphere as well as on its perceived depth. On the other hand, if the process that organizes the rotating spheres behaves like the form-independent apparent-movement process studied by Kolers and Pomerantz (1971), then a dissimilarity between corresponding elements in successive frames of the simulation should have no effect on the coherence of the percept.

\section{Method}

Subjects. Nine subjects volunteered to participate in the experiment. All subjects reported that they had $20 / 20$ visual acuity. Five of the nine subjects had previously served in a similar experiment. The functions produced by the experienced subjects had the same appearance as those produced by the inexperienced subjects and therefore separate analyses were not conducted.

Stimuli. The maximum diameter of the simulated spheres in the present experiment subtended a visual angle of $2.5^{\circ}$. All the vectors within each sphere had one of three lengths, $10^{\prime}, 20^{\prime}$, or $40^{\prime}$ visual angle. The mean luminance of any sphere depended upon the length of the vectors that composed $\mathrm{it}^{1}:$ (1) for $40^{\prime}$-vector spheres, $.07 \mathrm{~cd} / \mathrm{m}^{2}$; (2) for $20^{\prime}$-vector spheres, $.04 \mathrm{~cd} / \mathrm{m}^{2}$; and (3) for $10^{\prime}$ vector spheres, $.03 \mathrm{~cd} / \mathrm{m}^{2}$. The values of the display parameters that were not varied experimentally were as follow: (1) vector numerosity, at 24 vectors, (2) number of frames per cycle of rotation, at 40 frames, (3) frame duration, at $100 \mathrm{msec}$, (4) ISI, at 15 msec, and $(5)$ number of complete rotations per trial, at two. 
Procedure. The viewing conditions of the present experiment were the same as those described for Experiment 1. Each subject served in eight sessions, one practice session and seven experimental sessions, each held on a different day. During a single experimental session, a subject viewed one block of 30 different displays resulting from the factorial combination of two projection distances ( 1.2 and 6.0 sphere radii), three vector lengths $\left(10^{\prime}, 20^{\prime}\right.$, and $40^{\prime}$ ), and five levels of orientation difference between the vectors of successive frames $\left(0^{\circ}, 10^{\circ}, 25^{\circ}, 45^{\circ}\right.$, and $65^{\circ}$ from vertical). No feedback was provided after a trial. About $30 \mathrm{sec}$ elapsed between successive trials.

\section{Results and Discussion}

Rotation-direction judgments. Figures 3 and 4 show, for the 6.0 and 1.2 sphere-radii projection distances, respectively, the percentage of correct rotation-

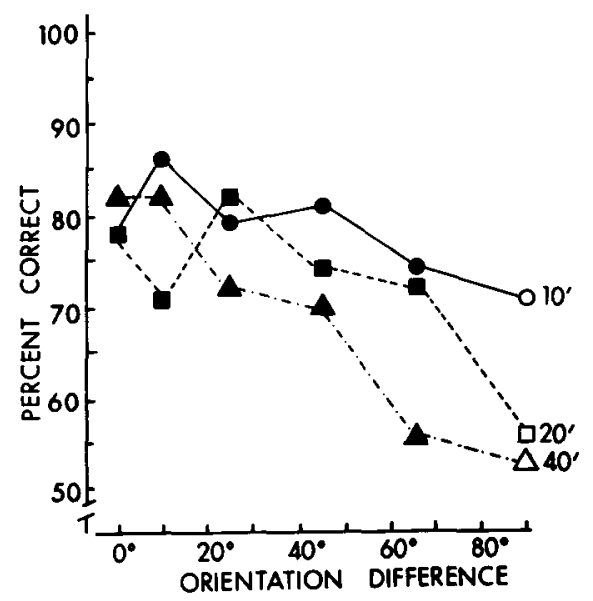

Figure 3. Percentage of correct rotation-direction judgments, for the 6.0 sphere-radii projection distance only, as a function of the orientation difference between the vectors in alternate frames of the display. Each curve depicts the effect for a different vector length (in minutes of visual angle).

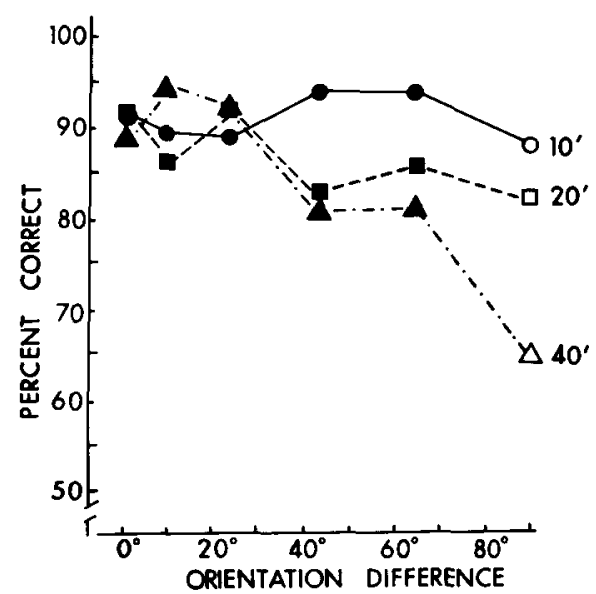

Figure 4. Percentage of correct rotation-direction judgments, for the 1.2 sphere-radii projection distance only, as a function of the orientation difference between the vectors in alternate frames of the display. Each curve depicts the effect for a different vector length (in minutes of visual angle). direction judgments as a function of the orientation difference between the lines of successive frames. The separate functions in each figure show the percentage associated with each of three line lengths. The first finding of interest was that the overall percentage of correct rotation-direction judgments was greater for the shorter simulated projection distance (Figure 4) than for the longer simulated projection distance (Figure 3), irrespective of line length or orientation difference. This finding is consistent with the results of Experiment 1, which showed that shorter simulated projection distances yield greater percentages of correct rotation-direction judgments than longer simulated projection distances. Secondly, increases in orientation difference resulted in decreases in the percentage of correct rotation-direction judgments. This effect was shown to be significant in a repeated measures analysis of variance $[F(4,32)=$ $3.54, \mathrm{p}<.025, \mathrm{SE}=1.83$ ]. Because most of the functions showed fairly sharp declines at orientation differences of $25^{\circ}$ and greater, a supplementary study was conducted to determine whether orientation differences of $90^{\circ}$ would lead to further reductions in rotation-judgment accuracy beyond those shown for the $65^{\circ}$ orientation differences. Seven experienced observers, four of whom had served in the main experiment, participated in the supplementary study. This study was conducted like the main experiment, with the exception that all of the data were collected from each subject in a single session with repeated randomized blocks of trials. The data from the supplementary study, averaged over the seven observers, are represented by the open symbols in Figures 3 and 4. Both figures show a continued decline in rotation-direction accuracy with increasing orientation differences.

In addition to the main effect of orientation difference, there was a significant effect of line length $[\mathrm{F}(2,16)=3.84, \mathrm{p}<.05, \mathrm{SE}=1.46$ (data from the supplementary study not included)]. The mean correct percentages for each line length (collapsed over orientation difference and projection distance) were $85.39 \%\left(10^{\prime}\right.$ vectors $), 81.42 \%\left(20^{\prime}\right.$ vectors $)$, and $79.83 \%$ ( $40^{\prime}$ vectors $)$. There was also a significant interaction between orientation difference and line length $[F(8,64)=2.63, p<.025, \mathrm{SE}=3.06]$. This interaction is important theoretically, because it shows that a given orientation difference does not have a constant effect on rotation-direction judgments. Rather, the effect of an orientation difference does depend upon the length of the lines being compared over time.

The results of Frisby and Julesz (1975) suggest that it may be the magnitude of the visual subtense between the endpoints of successive partner vectors that affected the rotation-direction judgments. This distance in three-dimensional space will be referred to 
LINE LENGTHS
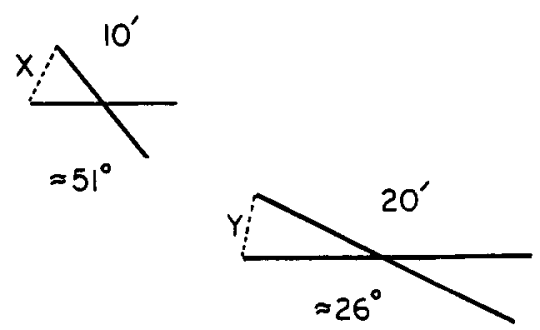

ORIENTATION DIFFERENCES

$$
X=Y
$$

Figure 5. Endpoint disparity. The distance between the endpoints of the vectors (in three-dimensional space) presented in alternate frames of the displays depends upon both the lengths of the vectors and the relative orientations of the vector pairs. In the example shown, in order to maintain a constant endpoint disparity (i.e., in order to keep $X=Y$ ) for vector pairs of different lengths $\left(10^{\prime}\right.$ vs. $20^{\prime}$ visual angle), the relative orientation of the vectors in the pairs must differ $\left(51^{\circ}\right.$ vs. $26^{\circ}$, respectively).

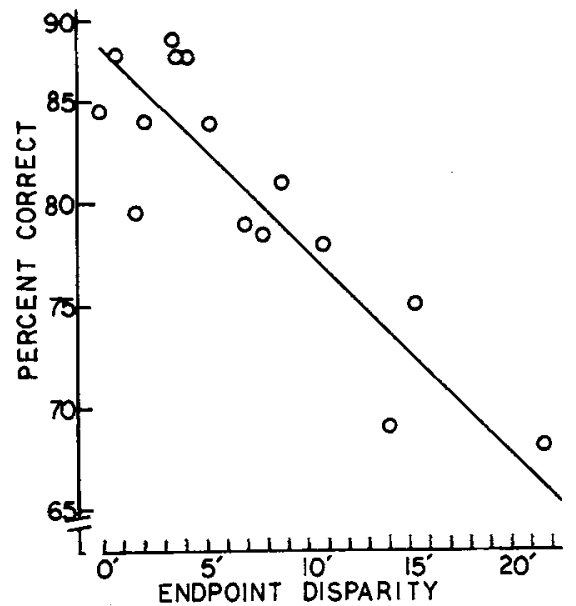

Figure 6. Percentage of correct rotation-direction judgments, collapsed over projection distance, as a function of endpoint disparity (in minutes of visual angle). The fitted line is the least squares regression line, $Y^{\prime}=\mathbf{- 1 . 0 0 X}+\mathbf{8 7 . 7}$.

as "endpoint disparity." It is shown in Figure 5 that in order to maintain a constant endpoint disparity with successive vector pairs of different lengths, the vectors in each pair must be oriented at different angles. Thus, for the two pairs shown in Figure 5, endpoint disparity is constant, i.e., $X=Y$. The interaction of orientation difference and line length was therefore analyzed by calculating the endpoint disparity, in terms of its visual subtense on the retina, for each line length and orientation difference. The original data from the main experiment were then entered into a two-way repeated measures analysis of variance with endpoint disparity and projection distance as factors. The main effect of projection distance was again significant, as was the main effect of endpoint disparity $[F(12,96)=3.60, p<.001]$. The relationship between endpoint disparity and percentage of correct rotation-direction judgments is shown in Figure 6. There it can be seen that percent correct rotation-direction judgments declines with increases in endpoint disparity. Also shown in Figure 6 is the regression line relating the two varibles. This line has a slope of -1 , and the linear correlation between rotation-direction accuracy and endpoint disparity (including the supplementary data) was $r=$ -.92 .

Depth judgments. The effect of the three independent variables on perceived depth were similar to their main effects on perceived rotation direction. That is, perceived depth decreased monotonically (and significantly) with increases in line length, with increases in orientation differences, and with an increase in projection distance. Table 1 shows the mean depth ratings obtained at each level of each variable, collapsed over the remaining variables. Again, there was a significant interaction of line length and orientation difference. Therefore, all data points from the main experiment were plotted as a function of endpoint disparity. Figure 7 shows these data and their regression line. As with the results of Frisby and Julesz (1975) for binocular vertical disparity, the present finding was that perceived depth declined steadily with increases in endpoint disparity. In fact, there was a significant linear correlation of endpoint disparity and mean depth ratings, $r=$ -.92 . The regression line has a slope of -.14 .

The present findings suggest that the process that is responsible for the perceptual pairing of corresponding elements in successive frames of rotation simulation is sensitive to the disparity between endpoints of partner vectors in successive frames. In this respect, the process responsible for organizing the display elements into a coherent percept responds to the details of the sphere elements rather than to their global, i.e., low spatial frequency, characteristics.

Table 1

Mean Depth Ratings Obtained at Each Level of Each Independent Variable in Experiment 2

\begin{tabular}{|c|c|c|c|c|c|c|c|c|c|}
\hline \multicolumn{5}{|c|}{ Orientation Difference (Degrees) } & \multicolumn{3}{|c|}{$\begin{array}{l}\text { Line Length } \\
\text { (Minutes Visual Angle) }\end{array}$} & \multicolumn{2}{|c|}{$\begin{array}{c}\text { Projection Distance } \\
\text { (Sphere Radii) }\end{array}$} \\
\hline 0 & 10 & 25 & 45 & 65 & 10 & 20 & 40 & 1.2 & 6.0 \\
\hline 4.86 & 3.91 & 3.69 & 3.23 & 2.97 & 4.81 & 3.64 & 2.75 & 4.07 & 3.40 \\
\hline
\end{tabular}




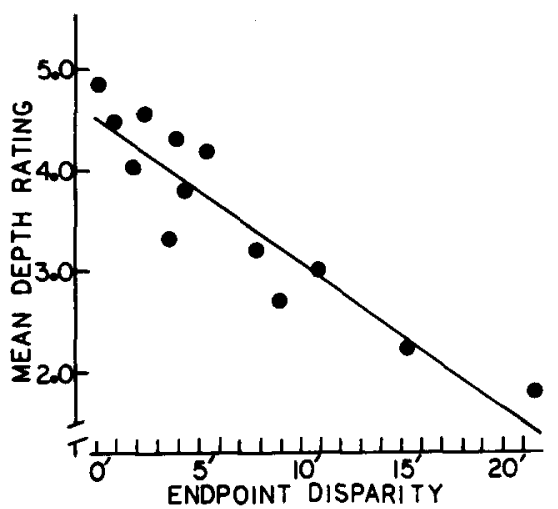

Figure 7. Mean depth ratings, collapsed over projection distance, as a function of endpoint disparity (in minutes of visual angle). The fitted line is the least squares regression line, $Y^{\prime}=\mathbf{- 0 . 1 4 X}$ +4.5 .

Physiological mechanisms sensitive to details like line length and line orientation are known to exist in the mammalian visual cortex (Hubel \& Wiesel, 1962). No doubt the present data can be accounted for in feature analytic terms.

\section{GENERAL DISCUSSION}

Experiments 1 and 2 were similar inasmuch as the manipulation of some variable (percentage of signal dots or orientation differences) was intended to make the task of perceptually "pairing" signal elements in successive frames of a simulation more difficult. To some extent, both manipulations were successful. However, it should be noted that although each manipulation produced a significant decrease in rotation-direction accuracy and in rated depth, the phenomenal experience of most subjects was not greatly affected by these manipulations until the level of noise was fairly extreme. That is, most of the time subjects reported that the displays appeared spherelike despite adverse stimulus conditions. The subjective impressions of the subjects were confirmed by their performance on more objective tasks. For example, as Figure 1 shows, rotation direction was correctly discriminated (about three standard errors above $50 \%$ ) when only $9 \%$ of the display elements carried the perspective signal in the high-perspective simulations. Similarly, in Experiment 2 rotationdirection discrimination rarely dropped below $70 \%$. Furthermore, subjects rarely used depth ratings lower than 2 in either Experiment 1 or Experiment 2. Thus, even though signal/noise ratio and endpoint disparity had significant effects, they were generally not able to eliminate the ability of the perceptual process to detect dynamic perspective information and construct coherent three-dimensional percepts.

The phenomenon under consideration also supports the proposal of Braddick (1974) and Petersik, Hicks, and Pantle (1978) that local apparent-movement interactions can serve as inputs to a higher-order figural organization process. That is, when described objectively, the present displays consisted of no more than a set of 45 dots or 24 vectors, each of which changed its position on a two-dimensional surface from time to time. If asked to, any observer could focus on an individual dot and attend to its apparent movement back and forth across the display screen. In such a case, the probability of reporting a sphere or rotation in depth would be greatly reduced. Yet, when an observer attends to all of the dots at once, the percept of a three-dimensional object emerges. The local movements of individual dots and the relationship among those dots over time are the vehicles whereby global dynamic perspective information is transmitted and whereby a coherent percept is constructed. Clearly, conclusions about the ability of observers to maintain coherent percepts should not be based solely upon performance in tasks that use only nonperspective displays or static displays.

One of the primary conclusions drawn from the present experiments, namely that even under adverse stimulus conditions dynamic perspective transformations yield stable percepts of three-dimensional structure, has been supported in an experiment by Lappin, Doner, Kottas, and Harris (Note 2). Lappin et al. used a procedure similar to that described here to construct two-frame simulations of spheres rotating through angles less than $360^{\circ}$. Surprisingly, as long as little or no noise was present in the displays, subjects could accurately determine rotation direction. However, with the two-frame displays, the introduction of even small amounts of noise nearly totally destroyed the global organization of the threedimensional percept.

The present experiments also bear some relationship to previous psychophysical studies that have used somewhat different displays. For example, with random-dot displays of movement in only two dimensions and in which some, but not all, display elements were correlated and shifted horizontally from frame to frame, Lappin and Kottas (Note 3) found that movement-direction discrimination increased significantly with increases in the number of frames in the display over the range of two to eight frames. A comparison of the results of Experiment 1 with those of Lappin and Kottas suggests that, in some sense, the sensitivity of the visual system to rotation movement in noise may be greater than its sensitivity to two-dimensional movement in noise, since a change by a factor of five in the number of frames in the three-dimensional displays of Experiment 1 did not significantly improve or hinder the discrimination of rotation direction. 
On the other hand, the results of Experiment 1 are consistent with Lappin and Kottas' finding that direction discrimination improved as the percentage of correlated elements between frames increased from $12.5 \%$ to $37.5 \%$. Their findings are comparable to those of the higher perspective conditions of the present experiments.

The importance of perspective in the specification of three-dimensional correlations (i.e., the perceptual "pairing" of corresponding elements, either in space, as in stereopsis, or in time and space, as in the present displays) cannot be overemphasized. For example, Gulick and Lawson (1976) conducted a study on three-dimensional object constancy in binocular vision. Their technique was to present a Julesz-type random-dot frame to each of the two eyes of an observer. In one of the frames, a small central region had been shifted horizontally by some amount to produce a binocular disparity between the projections of that region in the two eyes. As Julesz (1971) had previously shown, the binocular disparity of the central region resulted in the perception of a square separated from the background in depth. Gulick and Lawson extended that result by varying the percentage of elements in the central region that were actually shifted horizontally in the second of the two random-dot frames. They discovered that by the time as few as $20 \%$ of the dots were not shifted (i.e., $80 \%$ were shifted), the percept of the square in depth had broken down. On the other hand, in Experiment 1 with dynamic perspective information, the percept of an object in depth was maintained in the high perspective condition with only $9 \%$ of the elements undergoing the perspective transformation and $91 \%$ of the elements moving about randomly. This data point, shown in Figure 1, lies about three standard errors above the chance level of responding. To the extent that the two experiments can be compared, it is possible that the greater sensitivity of the observer to the simulated rotating sphere than to the stereoscopic stimuli of Gulick and Lawson can be attributed to the salience of dynamic perspective information.

The results of the present experiments are certainly consistent with Johansson's (1977) claim that, whenever possible, the visual system organizes transformations of visual scenes so as to preserve the perception of three-dimensional object constancy. In some respect, the information contained in dynamic three-dimensional perspective projections, and the corresponding preservation of object constancy, might be ecologically more significant for the human visual system than simple two-dimensional movement in the picture plane. As a further step in the understanding of object constancy, experiments directly comparing stereoscopic targets, two-dimen- sional movements, and three-dimensional movements need to be conducted.

\section{REFERENCE NOTES}

1. Petersik, J. T. Factors controlling the judgments of rotation direction and depth in certain dynamic perspective transformations. Unpublished doctoral dissertation, Miami University, 1978.

2. Lappin, J. S., Doner, J., Kottas, B. L., \& Harris, C. S. Sufficient conditions for detection of structure and motion in three dimensions. Paper presented at the annual meeting of the Psychonomic Society, San Antonio, November 1978.

3. Lappin, J. S., \& Kottas, B. L. Spatial and temporal linearity in detecting coherent structure in dynamic random-dot patterns. Paper presented at the annual meeting of the Association for $\mathbf{R e}$ search in Vision and Ophthalmology, Sarasota, Florida, April 1977.

\section{REFERENCES}

Bradbick, O. A short-range process in apparent movement. Vision Research, 1974, 14, 519-527.

BraUnSteIN, M. L. Depth perception through motion. New York: Academic Press, 1976.

EPSTEIN, W. (Ed.), Stability and constancy in visual perception. New York: Wiley, 1977.

Frisby, J. P., \& Julesz, B. The effect of orientation difference on stereopsis as a function of line length. Perception, 1975, 4, 179-186.

GiBson, J. J. Optical motions and transformations as stimuli for visual perception. Psychological Review, 1957, 64, 288-295.

Gulick, W. L., \& Lawson, R. B. Human stereopsis. New York: Oxford University Press, 1976.

Hubel, D. H., \& WIEseL, T. N. Receptive fields, binocular interaction and functional architecture in cat's visual cortex. Journal of Physiology, 1962, 160, 106-504.

Johansson, G. Perspective transformations as determining visual space perception. In R. B. MacLeod \& H. L. Pick (Eds.), Perception: Essays in honor of James J. Gibson. Ithaca, New York: Cornell University Press, 1974.

Johansson, G. Spatial constancy and motion in visual perception. In W. Epstein (Ed.), Stability and constancy in visual perception. New York: Wiley, 1977.

JuLEsz, B. Foundations of cyclopean perception. Chicago: University of Chicago Press, 1971.

Kolers, P. A., \& Pomerantz, J. R. Figural changes in apparent motion. Journal of Experimental Psychology, 1971, 73, 2-21.

Petersik, J. T., Hicks, K. I., \& Pantle, A. J. Apparent movement of successively generated subjective figures. Perception, $1978,7,371-383$.

\section{NOTE}

1. Although the luminance of these displays varied by only a small amount from trial to trial, a separate pilot study was conducted to determine whether the luminance of the spheres per se would influence the rotation-direction and depth judgments. A single highly practiced observer viewed 123 sequences of rotatingsphere simulations with no added noise. Each sphere was displayed at one of three intensities, ranging from slightly above threshold to well over a log unit above threshold. The differences in luminance produced no significant variations in the percentages of correct rotation-direction judgments, or in the values of the depth ratings.

(Received for publication September 21, 1978; revision accepted January $17,1979$. 